CAS CLINIQUE

\title{
Fibrodysplasie ossifiante progressive : à propos d'un cas et revue de littérature
}

Fibrodysplasia ossificans progressiva: a case report and literature review

Amina CHENTOUF

Faculté de médecine d'Oran

Université Oran1

Auteur correspondant : a.chentouf@atrss.dz_Soumis le 30/07/2020; accepté 10/09/2020 ; publié le 21/12/2020

\section{Citation: CHENTOUF, A. Fibrodysplasie ossifiante pro- gressive : à propos d'un cas et Revue de littérature.(2020) J Fac Med Or 4(2):619-624. \\ DOI:10.5281/zenodo.4282495 \\ MOTS CLÉS \\ Fibrodysplasie ossifiante progressive, FOP,ACVR1}

\section{Résumé}

Introduction

La fibrodysplasie ossifiante progressive (FOP) est une maladie génétique extrêmement rare, caractérisée par une ossification hétérotopique des tissus musculaires et conjonctifs évoluant par poussées, pouvant conduire à la création d'un véritable deuxième squelette. La rareté de cette maladie est à l'origine d'une errance diagnostique de plusieurs années. La FOP est décrite essentiellement chez le jeune enfant, les cas à début tardif sont extrêmement rares.

\section{Observation}

Nous rapportons un cas de Fibrodysplasie ossifiante progressive à expression tardive avec une revue de littérature des aspects cliniques, radiologiques, génétiques, évolutifs et thérapeutiques.

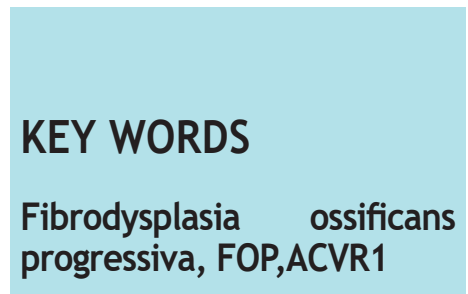

\section{Abstract}

Introduction

Fibrodysplasia Ossificans Progressiva (FOP) is an extremely rare genetic disease characterized by heterotopic ossification of muscles and connective tissue occurring in relapses, which can lead to the creation of a real second skeleton. The rarity of this disease is responsible for a delay in diagnosis of several years. FOP is described mainly in young children; cases with a late onset are extremely rare.

\section{Observation}

We report a case of late-onset Fibrodysplasia Ossificans Progressiva and we discuss clinical, radiological and genetic aspects, as well as treatment and outcomes. 


\section{Introduction}

La fibrodysplasie ossifiante progressive (FOP : OMIM 135100) est une maladie génétique extrêmement rare, caractérisée par une ossification hétérotopique des tissus musculaires et conjonctifs évoluant par poussées aboutissant à une douloureuse métamorphose en « homme de pierre » [1]. Bien qu'il s'agisse d'une maladie rare, ses caractéristiques cliniques sont singulières et pathognomoniques [2]. Elle est due à une mutation du gène ACVR1 (Activin Receptor IA) qui code pour une protéine morphogénétique [3]. La découverte de cette anomalie génétique a lancé de nombreux travaux de recherche permettant d'appréhender les bases physiopathologiques et d'aborder des pistes thérapeutiques spécifiques. La rareté de cette maladie est à l'origine d'une errance diagnostique de plusieurs années. La FOP est décrite essentiellement chez le jeune enfant, la survenue des symptômes à l'âge adulte est rare [4]. A travers cet article, nous rapportons le cas d'une patiente atteinte de FOP à expression tardive et nous discutons les aspects cliniques, radiologiques, génétiques, évolutifs et thérapeutiques de cette maladie.

\section{Observation}

Melle B.M.F., âgée de 37 ans, s'est présentée en consultation pour une contracture douloureuse des muscles du cou et de la mâchoire évoluant depuis 05 jours, limitant les mouvements de la tête et gênant la parole et la mastication.L'interrogatoire n'a révélé aucun antécédent personnel pathologique, aucune notion de consanguinité parentale et aucun cas similaire dans la famille.

L'histoire de la maladie remonte à l'âge de 20 ans par des épisodes d'enraidissement progressif du tronc et des membres évoluant par poussées. Ces épisodes étaient le plus souvent déclenchés par des microtraumatismes (mouvements brusques de la tête et du cou, chutes, injections intramusculaires) ou par des gestes invasifs (biopsie musculaire réalisée dans le cadre du bilan étiologique de sa maladie). L'examen clinique a mis en évidence une raideur cervicale, une hypertrophie douloureuse du muscle sternocléidomastoïdien gauche, une scoliose dorsale, des rétractions tendineuses aux quatre membres avec attitude en semi-flexion.

Nous avons relevé également une tuméfaction indurée au niveau du bras droit mesurant $40 \times 35 \mathrm{~mm}$, et une limitation de l'ouverture de la bouche gênant la mastication. Par ailleurs, elle présentait une déformation en hallux valgus des gros orteils qui étaient courts et de forme grossièrement triangulaire. L'ankylose des membres était à l'origine d'une impotence fonctionnelle invalidante avec impossibilité de s'asseoir et difficulté à la marche.
Le bilan biologique a objectivé un syndrome inflammatoire avec accélération de la vitesse de sédimentation des hématies, une élévation de la C ReactiveProtein (CRP) ainsi qu'une augmentation des phosphatases alcalines.

La radiographie du bras droit a montré une ossification importante du biceps brachial (Figure 1) ; et celle du pied a mis en évidence une ankylose inter phalangienne avec agénésie d'une phalange du gros orteil (Figure 2). Les radiographies du rachis cervical ont révélé une raideur avec aspect particulier des vertèbres plus hautes que larges ( $\mathrm{Fi}$ gure 3).

\section{Figure 1.Ossification hétérotopique du muscle biceps}

brachial droit

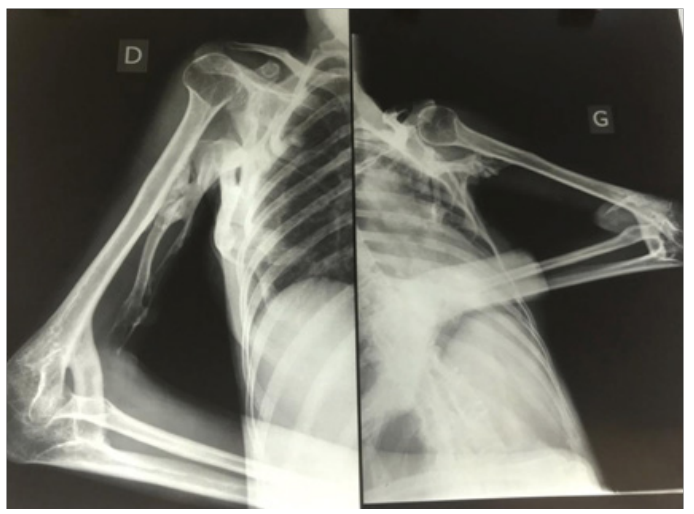

Figure 2. Maformation du gros orteil

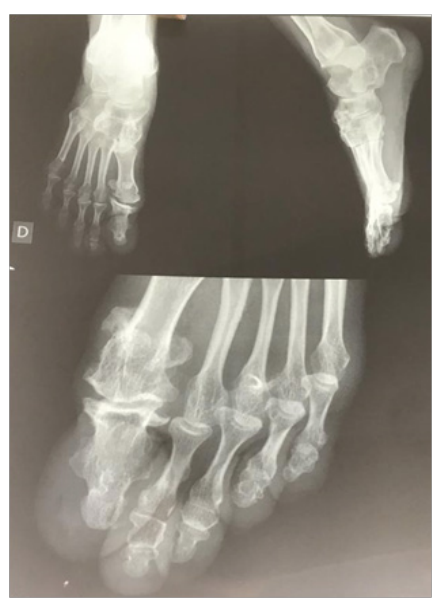

Devant ces éléments cliniques et radiologiques suggestifs, le diagnostic de FOP a été évoqué et une étude génétique a été réalisée après consentement de la patiente. Le séquençage a mis en évidence une mutation c.617G>A [p.Arg206His] à l'état hétérozygote, située dans l'exon 6 du gène ACVR1, confirmant ainsi le diagnostic de FOP.

La patiente a bénéficié d'un traitement symptomatique à base d'analgésiques et de prednisolone à raison $\mathrm{d}^{\prime} 1 \mathrm{mg} / \mathrm{K} / \mathrm{j}$ pendant 3 jours avec décroissance posologique sur 10 jours 
Figure 3. Vertèbres cervicales plus hautes que larges

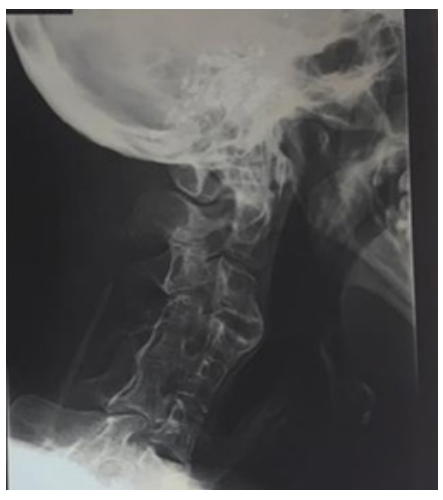

L'évolution fut marquée par une involution de la tuméfaction cervicale dès le 3ème jour de traitement, une disparition de la douleur, et une mobilité partielle de l'articulation temporo-mandibulaire ayant permis une restauration de la fonction masticatoire.

Un conseil génétique a été proposé avec évaluation de tous les membres de la famille à la recherche de signes évocateurs tels que les déformations des hallux.

La patiente a été instruite pour éviter tous les facteurs pouvant déclencher des poussées et aggraver le pronostic.

\section{Discussion}

Décrite pour la première fois par Guy Patin en 1692 [2], la fibrodysplasie ossifiante progressive également appelée maladie de Munchmeyer ou maladie de l'homme de pierre,est une affection extrêmement rare, affectant une personne sur deux millions [1]. Elle s'exprime le plus souvent chez le jeune enfant et touche indifféremment les deux sexes [4].

\section{1- Caractéristiques cliniquesde la FOP}

La FOP est une affection invalidante et progressive, caractérisée par des malformations congénitales des gros orteils et une formation osseuse extra-squelettique qui recouvre progressivement le squelette d'origine, entraînant une restriction des mouvements, une rigidité et le décès par complications cardiorespiratoires vers la quatrième décennie de vie [5].

Les individus naissent généralement en bon état de santé mais les anomalies des gros orteils sont presque toujours présentes à la naissance. Il s'agit notamment de gros orteils courts et déformés en valgus [6].

Durant les premières années de vie, des épisodes intermittents de gonflements douloureux des tissus conjonc- tifs et musculaires se produisent et évoluent en lésions osseuses indurées qui caractérisent la maladie. Alors que certaines poussées régressent spontanément, la plupart d'entre elles transforment les tissus mous (aponévroses, fascia, ligaments, tendons et muscles squelettiques) en os hétérotopique mature par un processus d'ossification endochondrale qui conduit à une immobilité permanente.

L'ossification hétérotopique commence d'abord dans les régions cervicales, paravertébrales et les épaules, puis gagne les hanches et les membres. Elle progresse d'une manière typique cranio-caudale, postéro-antérieure et proximo-distale, mimant le développement normal du squelette embryonnaire [7]. Certains muscles tels que le diaphragme, la langue, les muscles oculomoteurs, le myocarde et les muscles lisses sont épargnés.

Les traumatismes mineurs tels que les injections intramusculaires, des ecchymoses, des chutes ou des maladies virales telles que la grippe peuvent déclencher de nouvelles poussées douloureuses de FOP conduisant à une ossification hétérotopique progressive [2]. Par ailleurs la biopsie ou la chirurgie de l'os hétérotopique conduit de façon inéluctable à une aggravation du processus d'ossification avec apparition de nouvelles formations osseuses et des calcifications comme cela a été observé chez notre patiente [8].

En dépit de cette présentation clinique typique, les délais de diagnostic sont longs pouvant aller jusqu'à deux décennies. Chez notre patiente, le diagnostic a été posé 17 ans après le début des symptômes. Cette errance diagnostique serait due à la rareté de la maladie. En effet, la FOP est souvent diagnostiquée à tort comme une fibromatose juvénile agressive, un lymphœdème ou un sarcome des tissus mous. Une enquête réalisée sur les cinq continents a montré des diagnostics erronés dans environ $90 \%$ des cas, $67 \%$ des patients étant soumis à des procédures de diagnostic invasives potentiellement dangereuses, aggravant la progression de la maladie [9].

\section{2- Evolution de la maladie}

L'ossification est épisodique, mais le handicap est cumulatif. La plupart des patients atteints de FOP sont confinés dans un fauteuil roulant à la troisième décennie de leur vie et ont besoin d'une assistance à vie pour accomplir les activités de la vie quotidienne. Une perte de poids importante peut résulter d'une ankylose de la mâchoire. La pneumonie ou l'insuffisance cardiaque droite peuvent compliquer la rigidité de la paroi thoracique. L'âge médian de survie est d'environ 40 ans, et la mort survient le plus souvent dans le cadre de complications cardio-respiratoires [5].

\section{3- Aspects radiologiques de la FOP}

Sur le plan radiologique, l'exploration du rachis cervical met en évidence un aspect allongé et étroit avec fusion des facettes articulaires des vertèbres C2 à C7 avant 
même l'apparition des premières poussées. Les radiographies des pieds montrent un monophalangisme du premier orteil avec aspect trapu et élargi du premier métacarpe. La première phalange est courte, de forme triangulaire, parfois fusionnée avec le premier métatarsien ou avec la seconde phalange [9]. Les radiographies des muscles atteints montrent une ossification sous forme d'images opaques (Figure).

\section{4- Etiopathogénie}

La FOP est associée à une mutation du gène ACVR1, qui code pour un récepteur aux BMP (Bone Morphogenetic Protein) $1 \mathrm{~A}$, également appelé activine-like kinase 2 (ALK2). La mutation récurrente c.617G >A (p.Arg206His) mise en évidence chez notre patiente est retrouvée chez la majorité des patients présentant une forme classique de FOP [3]. Cette anomalie est responsable d'une activation de l'ostéogenèse dans les sites ectopiques sans liaison au ligand. De rares cas familiaux ont également été décrits avec un mode de transmission autosomique dominant [3].

L'implication du système immunitaire dans la survenue des ossifications hétérotopiques est indéniable. En effet, la présence de macrophages, de lymphocytes et de mastocytes dans les phases précoces de poussées de la maladie, le lien entre les poussées et les épisodes d'infections virales, ainsi que la régression des poussées sous corticostéroïdes, soutiennent l'implication du système immunitaire inné dans la pathogenèse des lésions de FOP.

\section{5- Prise en charge thérapeutique}

A ce jour, il n'existe aucun traitement spécifiquede la maladie. Le respect des mesures préventives et le traitement symptomatique restent la seule option thérapeutique.

\section{5-1- Mesures préventives:}

Les facteurs sur lesquels il faut agir sont les gestes invasifs non indispensables, les chutes ainsi que les traumatismes. Pour cela, il faut préconiser le port de chaussures légères antidérapantes et l'adaptation du domicile avec éviction des obstacles. Les injections intramusculaires sont proscrites dès que le diagnostic est confirmé.

Les soins réguliers de la cavité buccale sont essentiels pour prévenir des complications infectieuses. Les anesthésies peuvent être réalisées par micro-injections interligamentaires [10].

$\mathrm{Au}$ fur et à mesure que l'os hétérotopique s'accumule, l'amplitude des mouvements est progressivement perdue, conduisant à une immobilité presque complète. Les approches de réadaptation actuelles et futures devraient être axées sur l'amélioration des activités de la vie quotidienne.

\section{5-2- Traitement des poussées :}

Les corticostéroïdes sont proposés dans les poussées impliquant les grosses articulations et la mâchoire [10]. Ils doivent être administrés précocement et à fortes doses pendant 4 jours (Prednisone 1 à $2 \mathrm{mg} / \mathrm{K} / \mathrm{j}$ ) avec dégression sur 10 à 15 jours. Les anti-inflammatoires non stéroïdiens peuvent être prescrits en relais aux corticoïdes pendant plusieurs semaines selon le contexte. Les inhibiteurs de la cyclo-oxygénase-2, les inhibiteurs des leucotriènes et les stabilisateurs des mastocytes pourraient être utiles dans la prise en charge de l'inconfort chronique et des poussées ; mais à ce jour, aucune thérapie n'a prouvé son efficacité pour modifier l'histoire naturelle de la maladie.

\section{Conclusion}

Etant une affection extrêmement rare mais invalidante, la FOP doit être évoquée devant toute hypertrophie musculaire non expliquée survenant à n'importe quel âge ; associée à des malformations congénitales des gros orteils. Le diagnostic précoce est primordial car il permet de ralentir la progression de la maladie et aide de manière cruciale à éviter les biopsies ou les procédures préjudiciables.La découverte du gène ACVR1 apporte l'espoir de développer des thérapies pour cette maladie, jusque là incurable.

\section{Conflits d'intérêt}

L'auteure déclare n'avoir aucun conflit d'intérêt. 


\section{Références bibliographiques}

[1] Kaplan FS, Le Merrer M, Glaser DL, et al. Fibrodysplasiaossificansprogressiva. Best PractResClinRheumatol 2008;22:191-205

[2] Pignolo JS, Shore EM, Kaplan FS: FibrodysplasiaOssificansProgressiva: Clinical and genetic aspects. Orphanet Journal of Rare Disease. $2011 ;(1) 6: 80$

[3] Shore EM. FibrodysplasiaOssificansprogressiva (FOP): A humangeneticdisorder of extraskeletalbone formation, or - How does one tissue becomeanother? WileyinterdiscipRevDevBiol. 2012; 1 (1): 153-65

[4] Bridges AJ, Hsu KC, Singh A, Churchill R, Miles J. Fibrodysplasia (myositis) ossificansprogressiva. SeminArthritisRheum. 1994; 24(3): 155-64

[5] Kaplan FS, Zasloff MA et al: EarlyMortality and Cardiorespiratoryfailure in patients withfibrodysplasiaOssificansprogressivaJBone Joint Surg Am 2010, 92:686-91

[6] Nakashima Y, Haga N, Kitoh H, et al. Deformity of the greattoe in fibrodysplasiaossificansprogressiva. J OrthopSci. 2010;15(6):804809

[7] Baujat G, Michot C, Le Quan Sang KH, Cormier-Daire V. Fibrodysplasia ossificans progressiva and progressive osseous heteroplasia. Rev Rhum Monog. 2019; 86(1):40-5

[8] Zan X, Wang J, You C: The danger of biopsy in fibrodysplasiaOssificansProgressiva. Arch Dis Child. 2012; 97 (9): 785-6

[9] Kitterman JA, Kantanie S, Rocke DM, Kaplan FS. IatrogenicHarmCaused by Diagnostic Errors in FibrodysplasiaossificansProgressiva. Paediatrics. 2005; 116 (5): 654-661

[10] Pignolo RJ, Shore EM, Kaplan FS.FibrodysplasiaOssificansProgressiva: Diagnosis, Management, and TherapeuticHorizons.PediatrEndocrinolRev. 2013; 10(02): 437-448 
